\title{
The succession pattern of soil microbial communities and its relationship with tobacco bacterial wilt
}

\author{
Jiaojiao Niu ${ }^{1,2+}$, Zhongwen Rang ${ }^{3 \dagger}$, Chao Zhang ${ }^{3}$, Wu Chen ${ }^{3}$, Feng Tian ${ }^{4}$, Huaqun Yin ${ }^{1,2^{*}}$ and Linjian Dai ${ }^{3 *}$
}

\begin{abstract}
Background: The interaction mechanism between crop and soil microbial communities is a key issue in both agriculture and soil ecology. However, how soil microbial communities respond to crop planting and ultimately affect crop health still remain unclear. In this research, we explored how soil microbial communities shifted during tobacco cultivation under different rotation systems (control, maize rotation, lily rotation and turnip rotation).

Results: Our analyses showed that soil microbial communities had a general response pattern to tobacco planting, as the abundances of Proteobacteria and Planctomycetes increased while Acidobacteria and Verrucomicrobia decreased during tobacco cultivation, no matter which rotation system was adopted. Notably, tobacco decreased the diversity and co-occurrence of soil microorganisms, but maize rotation might suppress tobacco bacterial wilt by alleviating the decrease in biodiversity and co-occurrence. Molecular ecological network analysis indicated that there was stronger competition between potential disease suppressive (e.g., Acidobacteria) and inducible bacteria (e.g., Chloroflexi) in maize rotation systems. Both soil properties (e.g., pH, Ca content) and microbial communities of tobacco mature period depended on their counterparts of fallow period, and all these factors shaped tobacco disease comprehensively.

Conclusions: Both soil microbial communities of fallow stage and tobacco selection shaped the communities of tobacco mature stage. And effective rotation crop (maize) could decrease the incidence of tobacco bacterial wilt by alleviating the decrease in diversity and co-occurrences of microbial populations. This study would deepen our understanding about succession mechanism of soil microbial communities during crop cultivation and their relationship with crop health.
\end{abstract}

Keywords: Soil microbial communities, Succession mechanism, Crop health, Illumina sequencing, Molecular ecological network

\section{Background}

Soil microbes are drivers of plant diversity and productivity in terrestrial ecosystems, while plants also affect soil physical and chemical environment as well as soil organisms. Unrevealing how soil microbial communities change in response to crop planting is a central issue in both ecology and agriculture [1]. Particularly, how

\footnotetext{
* Correspondence: yinhuaqun@gmail.com; linjianpaper@foxmail.com

${ }^{\dagger}$ Equal contributors

'School of Minerals Processing and Bioengineering, Central South University,

Changsha 410083, China

${ }^{3}$ College of agronomy, Hunan Agricultural University, Changsha 410128,

China

Full list of author information is available at the end of the article
}

pathogen populations response to crop planting is significant for exploring pathogenesis of crop disease. It has been suggested that disease inducible microorganisms originated from initial soil microbial communities before crop planting [2]. Researches in this area make it possible to control pathogen populations before they cause severe crop disease [3, 4], but few studies explore the relationship between succession of soil microbial communities and plant health.

However, the response of microbial communities to crop planting is often unpredictable and too variable. It is difficult to summarize a general response pattern, because a kind of crop (e.g., wheat, tobacco) could be planted in farmlands with different climates, soil 
properties and rotation systems, resulting in spatial and temporal variability of soil microbial communities. Some DNA-based studies indicated the importance of spatial variability in microbial communities [5, 6]. Meanwhile, other studies demonstrated the significance of temporal variability, so examining the effect of management practices on soil quality based on microbial communities should consider seasonal changes [7]. Due to these variations, studies about soil microbial communities of a same crop were usually not complied with each other. Take soil microbial communities of tobacco as an example, it was reported in a research that fungi and actinomycete had similar abundance pattern, and both of them descended at the early stages then ascended [8], while in another study fungi reached peak at rapid growth stage and the largest number of actinomycete appeared in rosette stage [9]. In a word, the spatial and temporal variabilities of microbial communities are ubiquitous, therefore such background noises must be excluded, at least taken into consideration, when exploring general response pattern of soil microbial communities to crop planting.

Analytical method is another limitation in investigating soil microbial communities. It has been known that interactions among microbial populations are ubiquitous [10], which provide a better understanding of the relationship between complexity and ecological stability. However, few studies involved interactions among microorganisms, except for several researches of plants pathogens and their antagonistic bacteria [11, 12]. Mainly based on experiments, these studies provided little information about complex interactions among different microbial populations, which might affect each other directly or indirectly. Random matrix theory (RMT) is powerful in identifying molecular ecological networks in microbial communities, and it has been employed well to analyze the co-occurrence/interaction among different microbial populations $[13,14]$. It provides us an opportunity to explore how co-occurrences/ interactions among different microbial populations change with plant growth and disease occurrence, which is an issue poorly studied in agriculture.

Tobacco is a typical continuous cropping intolerant crop, for continuous cropping usually induces serious crop disease, such as tobacco bacterial wilt and black shank $[15,16]$. Thus it usually be cultivated under different rotation systems (e.g., turnip, wheat, rice and maize). Studies about function mechanism of crop rotation have revealed that it is related to soil microbes, but how microbial communities shift after rotation crop planting and ultimately shape tobacco health still remain unclear $[16,17]$. These characteristics make tobacco a suitable subject to explore succession of soil microbial communities, especially pathogens, during crop planting. Tobacco has 4 important growth stages, including transplanting stage, rosette stage, fast-growing stage and mature stage. Mature stage is also the disease stage when soil microorganisms, at least pathogens, are most active. In order to reveal the response mechanism of soil microorganisms to tobacco planting and disease, we chose two key time points: the time before tobacco transplanting and the time during tobacco disease period. We hypothesized that (i) soil microbial communities had a general succession pattern from fallow period to tobacco mature period; (ii) rotation crops affected tobacco health through influencing soil microbial communities and their succession. Using 16S rRNA gene sequencing, we explored the general effects of tobacco planting on microbial community composition, structure and cooccurrence pattern across 4 different rotation types. As a result, we revealed the succession pattern of soil microbial communities during tobacco plating, and found that maize rotation could suppress tobacco bacterial wilt by alleviating the decrease in biodiversity and cooccurrences among bacterial populations.

\section{Methods}

\section{Sampling, Illumina sequencing and data processing}

Tobacco cultivated under different rotation systems was investigated, which were continuous tobacco cropping (Control), tobacco-maize rotation cropping (MR), tobacco-lily rotation cropping (LR), and tobacco-turnip rotation cropping (TR). Samples were collected from each field using checkerboard sampling method on March 27th (fallow period before tobacco planting) and July 28th (tobacco mature stage or diseased period) respectively. Field design, sampling, sample processing, measurement of soil properties, DNA extraction and $16 \mathrm{~S}$ rRNA gene sequencing were conducted following the methods described before [18]. All the 16S rRNA sequences were deposited in GenBank database and the accession numbers were KR831285 - KR855564. Dissimilarity tests were based on Bray-Curtis dissimilarity index using analysis of similarities (ANOSIM) [19]. Differences in abundances among 4 groups were determined by a one-way analysis of variance (ANOVA) followed by least significant difference (LSD) test [20]. Bacterial community diversity was calculated using Shannon-Weiner's $\mathrm{H}^{\prime}$ and evenness. Multivariate statistical analyses of sequencing data were conducted, including detrended correspondence analysis (DCA) for comparing the different microbial communities, as well as mantel test [21] and partial least squares path modeling (PLSPM) for linking microbial communities to environmental variables. All the analyses were performed in $\mathrm{R}$ v. 2.6.1 with the packages vegan and plspm [22] or online (http://ieg.ou.edu/). 


\section{Network construction and characterization}

As previously described, random matrix theory (RMT)based approaches were used for network construction $[14,23]$, hub and connector gene identification, and topological property determination with an automatic threshold. To ensure correlation reliability, OTUs in at least 5 out of 8 replicates were used for network analysis. Various network properties such as average degree, average path distance, average clustering coefficient and modularity index were characterized. The network modules were generated using rapid greedy modularity optimization.

The experimental data used for constructing phylogenetic molecular ecological networks (pMEN) were based on 16S rRNA gene sequencing analysis. First, a Pearson correlation matrix was constructed [24]. The correlation matrix was then converted to a similarity matrix, which measures the degree of concordance between the abundance profiles of OTUs across different samples by taking the absolute values of the correlation matrix [24, 25]. Subsequently, an adjacency matrix, which encodes the connection strength between each pair of nodes, was derived from the similarity matrix by applying an appropriate threshold, which was defined using the RMT-based network approach as previously described [23, 26, 27]. The Cytoscape 2.6.0 [28] software was used to visualize the network graphs. Other information about genes (e.g., taxonomy, relative abundance) and edge information (e.g., weights and positive and negative correlations) was also imported into the software and visualized in the network figures. Since we are interested in the temporal variability of network interactions, the pMENs were constructed separately based on sequencing data of Control, MR, LR and TR of 2 periods, respectively.

\section{Results}

\section{Soil geochemical properties}

A summary of soil properties, including soil $\mathrm{pH}$, water content and amount of $\mathrm{Ca}, \mathrm{K}, \mathrm{Mn}, \mathrm{Fe}, \mathrm{Co}, \mathrm{Cr}$ and $\mathrm{Ni}$, was described in Additional file 1: Table S1. Water content was significantly $(p<0.01)$ higher in fallow period (T-test) and showed no difference among 4 rotation groups. The value of $\mathrm{pH}$ ranged from 4.43 to 5.40, remained unchanged in MR and TR, but significantly ( $p$ $<0.05)$ higher in Control and lower in LR. But it showed no significant difference between 2 periods. ICP analysis revealed the total amount of various elements. Most of them were more abundant in fallow period (e.g. Fe and $\mathrm{Cr}$ ) except for $\mathrm{K}$, which were significantly $(p<0.05)$ higher in tobacco mature period. The amount of elements also showed difference among four rotation systems. For example, $\mathrm{K}$ and $\mathrm{Ni}$ were significantly $(p<0.05)$ less abundant in Control, as well as the amount of $\mathrm{Ca}$ was higher in MR. Besides, Control and TR had higher tobacco disease rate $(57.78 \%$ and $59.62 \%)$, while it was the lowest in MR (23.54\%).

\section{Overview of microbial community diversity}

After resample, we obtained 18000 high-quality 16S rRNA gene sequences per sample. Rarefaction curve showed that the numbers of OTUs were almost saturate in all samples, and enough for community analysis (Additional file 1: Figure S1). After clustering at $97 \%$ sequence identity, 13,911 OTUs were identified in this study, out of 120 OTUs were classified as archaea. Within the bacterial domain, 4 major microbial phyla accounted for $47.33 \%-59.67 \%$ of all reads (Additional file 1: Figure S2), and they were Proteobacteria (18.05\% - $28.86 \%)$, Acidobacteria (6.05\%, $23.44 \%)$, Actinobacteria (5.65 \%, $11.28 \%)$ and Chloroflexi (3.99 \%, $15.44 \%)$. And about $14 \%-22 \%$ of sequences were not assigned to any known phylum (Additional file 1: Figure S2). Microbial communities were more diverse at the genus level. The top five predominant microbial genera were Acidobacteria_Gp6 (0.44 \% - $11.83 \%)$, Ktedonobacter $(0.28 \%$ - $8.88 \%)$, Spartobacteria_genera_incertae_sedis (1.04 \% - $4.99 \%)$, Acidobacteria_Gp1 (0.62 \% - $6.82 \%)$ and Gemmatimonas (0.89 \% - $3.08 \%)$. But the most abundant genus was different in each group.

To evaluate the similarity of these microbial communities in structure, we conducted dissimilarity test and DCA. DCA graph showed that samples in fallow period were separated clearly from tobacco mature period, indicating that soil microbial communities shifted during tobacco cultivation (Fig. 1). Dissimilarity test showed that microbial community composition and structure of 4 crop rotation systems were significantly $(p<0.01)$ different from each other, no matter in fallow period or in

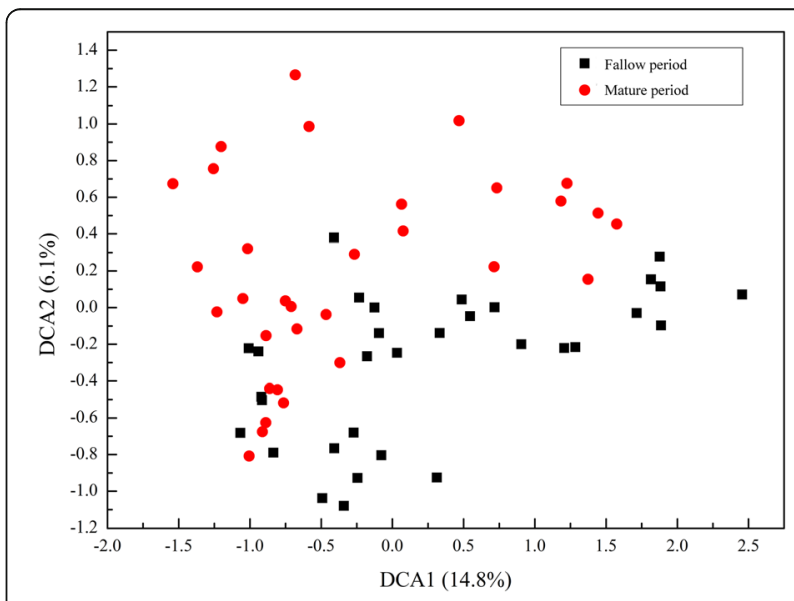

Fig. 1 Detrended correspondence analysis (DCA) of 16r RNA gene sequencing data for all 80 samples. The values of DCA1 and DCA2 are percentages of total variations that can be attributed to the corresponding axis 
mature period (Tables 1 and 2). Both the Shannon diversity and Pielou evenness indices decreased significantly $(p<0.05)$ in mature period, except for MR. For example, in LR the diversity index decreased from 6.26 to 5.50 and evenness index decreased from 0.82 to 0.76 (Table $3)$.

\section{Microbial communities shift from fallow to tobacco mature period}

Microbial community composition and structure were different in 4 rotation systems, such as Chloroflexi was less abundance in MR than in Control, and Acidobacteria was more abundant in TR than in Control (Fig. 2a). At the genus level, Acidobacteria_Gp6 and Acidobacteria_Gp4 were more abundant in MR while Ktedonobacter and Singulisphaera were more abundant in Control (Fig. 2b). However, most of the microbial populations had the similar abundance pattern in two periods. Pearson correlation analyses were conducted to evaluate the similarity in abundance pattern. Our results showed that relative abundances of Acidobacteria, Chloroflexi, Planctomycetes, Firmicutes, Nitrospira and BRC1 in fallow period were positively correlated with that in tobacco mature period. These phyla accounted for $33.73 \%-$ $52.26 \%$ of total population except for unclassified OTUs, but accounted for $28.33 \%-42.67 \%$ of total population including unclassified OTUs. Only the abundances of Actinobacteria showed negative correlation between 2 periods (Additional file 1: Table S2a). At the genus level, we found that 11 bacterial genera showed positive correlation in abundances between 2 periods, such as Ktedonobacter, Singulisphaera and Acidobacteria_Gp2 (Additional file 1: Table S2b). And no microbial genus showed negative correlation between 2 periods. In summary, direct correlations were found for a large percent of microbial populations, indicating that there was a general response pattern of soil microbial communities to tobacco cultivation across rotation types, and that soil microbial communities in mature period were shaped by the fallow microbial communities.

Table 1 Dissimilarity test of 165 rRNA gene sequencing data among four groups in fallow period and mature period respectively

\begin{tabular}{|c|c|c|c|c|c|c|c|}
\hline \multirow{2}{*}{$\begin{array}{l}\text { Sampling } \\
\text { time }\end{array}$} & \multirow[t]{2}{*}{ Group } & \multicolumn{2}{|c|}{ Control } & \multicolumn{2}{|l|}{$M R$} & \multicolumn{2}{|l|}{ LR } \\
\hline & & $\bar{D}$ & $P$ & $\bar{D}$ & $P$ & $\bar{D}$ & $P$ \\
\hline \multirow[t]{3}{*}{ Fallow period } & MR & 0.48 & $<0.01$ & & & & \\
\hline & $L R$ & 0.51 & $<0.01$ & 0.50 & $<0.01$ & & \\
\hline & TR & 0.52 & $<0.01$ & 0.50 & $<0.01$ & 0.54 & $<0.01$ \\
\hline \multirow[t]{3}{*}{ Mature period } & MR & 0.48 & $<0.01$ & & & & \\
\hline & LR & 0.46 & $<0.01$ & 0.47 & $<0.01$ & & \\
\hline & $\mathrm{TR}$ & 0.46 & $<0.01$ & 0.47 & $<0.01$ & 0.47 & $<0.01$ \\
\hline
\end{tabular}

$D$ bray-cutis distance, $P$ significance level
Table 2 Shannon diversity and Pielou evenness of soil microbial communities

\begin{tabular}{llllll}
\hline Index & Sampling time & Control & MR & LR & TR \\
\hline Shannon diversity & Fallow period & $6.42^{\mathrm{a}}$ & $6.36^{\mathrm{a}}$ & $6.26^{\mathrm{a}}$ & $6.43^{\mathrm{a}}$ \\
& Mature period & $6.08^{\mathrm{a}}$ & $6.50^{\mathrm{b}}$ & $5.52^{\mathrm{c}}$ & $5.91^{\mathrm{ac}}$ \\
& $P$ value & $<\mathbf{0 . 0 5}$ & 0.11 & $<\mathbf{0 . 0 5}$ & $<0.05$ \\
Pielou evenness & Fallow period & $0.83^{\mathrm{a}}$ & $0.81^{\mathrm{b}}$ & $0.82^{\mathrm{ab}}$ & $0.83^{\mathrm{a}}$ \\
& Mature period & $0.81^{\mathrm{ab}}$ & $0.82^{\mathrm{a}}$ & $0.77^{\mathrm{c}}$ & $0.79^{\mathrm{b}}$ \\
& $P$ value & $<\mathbf{0 . 0 5}$ & 0.23 & $<\mathbf{0 . 0 5}$ & $<0.05$
\end{tabular}

Significant differences $(p<0.05)$ between two periods are indicated in bold. And significant $(p<0.05)$ differences among four groups are labeled with alphabet

Actually, tobacco planting had certain impacts on soil microbial communities, with community composition and structure changed from fallow stage to tobacco mature stage. Generally, relative abundances of Proteobacteria and Actinobacteria showed a tendency of increase at tobacco mature stage. On the contrary, Chloroflexi and Acidobacteria were more abundant at fallow stage, especially in LR (Fig. 2a). Especially, Actinobacteria was more abundant in mature period than fallow period only in MR, but showed no significant difference between two periods in other three groups. At the genus level, Acidobacteria_Gp6 and Acidobacteria_Gp4 were more abundant at fallow stage, while the abundances of Singulisphaera were higher at mature stage (Fig. 2b).

\section{Co-occurrences among different microbial populations}

To understand the co-occurrence pattern among different microbial populations in 8 groups of microbial communities, 16S rRNA gene sequencing data were used to construct pMENs by RMT-based network approach. Major topological properties of 8 empirical pMENs showed that, with the same threshold (0.950), there were a lot more nodes and links in ecological networks of MR than other 3 groups no matter at fallow stage or tobacco mature stage (Additional file 1: Table S3). More importantly, the numbers of nodes and links decreased in tobacco mature period for networks of control, LR and TR, except for that of MR. The degree distributions in all constructed pMENs well fitted the power law model as linear correlations changed from 0.779 to 0.907 . For the average path distance, it decreased at mature stage in pMENs of all groups except for MR, suggesting that ecological networks might more closely connected at fallow stage (Additional file 1: Table S3). The same tendency was also seen from Additional file 1: Figure S3.

To explore the mechanism of how (potential) probiotic bacteria interacted with other microbial populations to protect plants from disease, we analyzed the sub-networks of Pseudomonas and Acidobacteria_Gp4, whose abundances were negatively correlated to tobacco disease rate. Because we were interested in the co-occurrences relationships which 

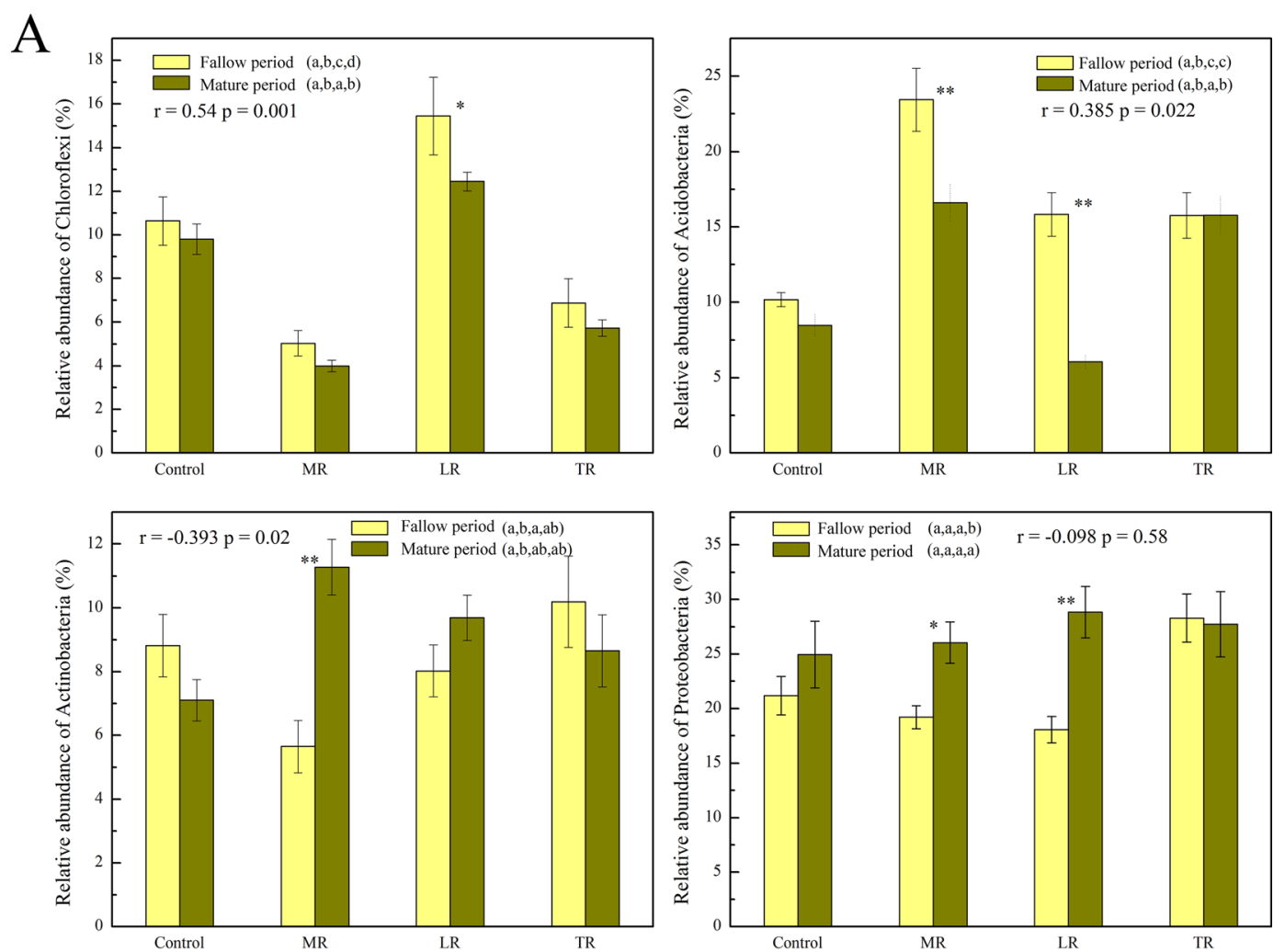

\section{B}
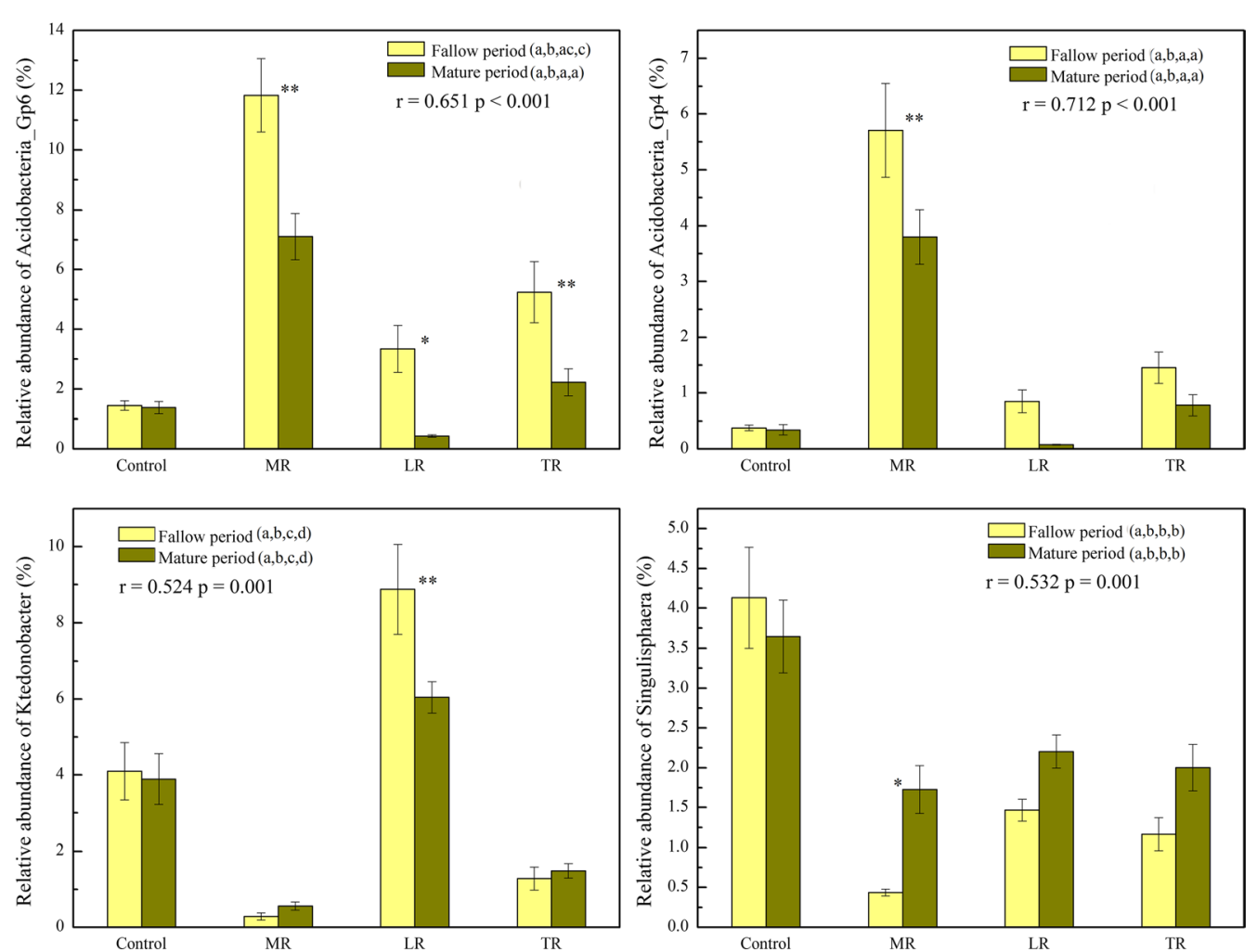

Fig. 2 Relative abundances of dominant phyla (a) and genera (b) of soil microbial communities in 8 groups. Correlations in abundance of microbial populations between 2 periods are indicated with $r$ and $p$ values. Significant differences $(p<0.05)$ among 4 groups are indicated with alphabet. Significant differences between 2 periods are labeled with ${ }^{*}\left({ }^{*} p<0.05,{ }^{*} p<0.01\right)$ 
were related to tobacco bacterial wilt directly, we only focused on the sub-networks of mature period when tobacco was severely diseased. Top 3 Gp4 OTUs with the highest connections were chosen to construct the sub-network of each group. Most of the links (69\%- $95 \%$ ) were negative in all 4 sub-networks. Most OTUs of Gp4 were negatively linked with OTUs of Proteobacteria, Chloroflexi, Actinobacteria and Plantomycetes. Of them, Proteobacteria and Actinobacteria had no significant correlation with tobacco disease rate, but the abundances of Chloroflexi and Plantomycetes were negatively correlated with tobacco disease rate. Of OTUs positively linked with Gp4, most of them were
Acidobacteria and Proteobacteria, and Acidobacteria were potential probiotic bacteria. Compared with other three networks, Gp4 in MR had more negative links with Chloroflexi and Planctomycetes, and more positive links with Acidobacteria (Fig. 3). It suggested that Gp4 enhanced their cooperation with potential probiotic bacteria and competition with pathogens in MR.

\section{Relationship among soil properties, microbial communities and tobacco health}

Tobacco disease was a key factor in shaping abundance patterns of many taxonomic groups. Based on Pearson

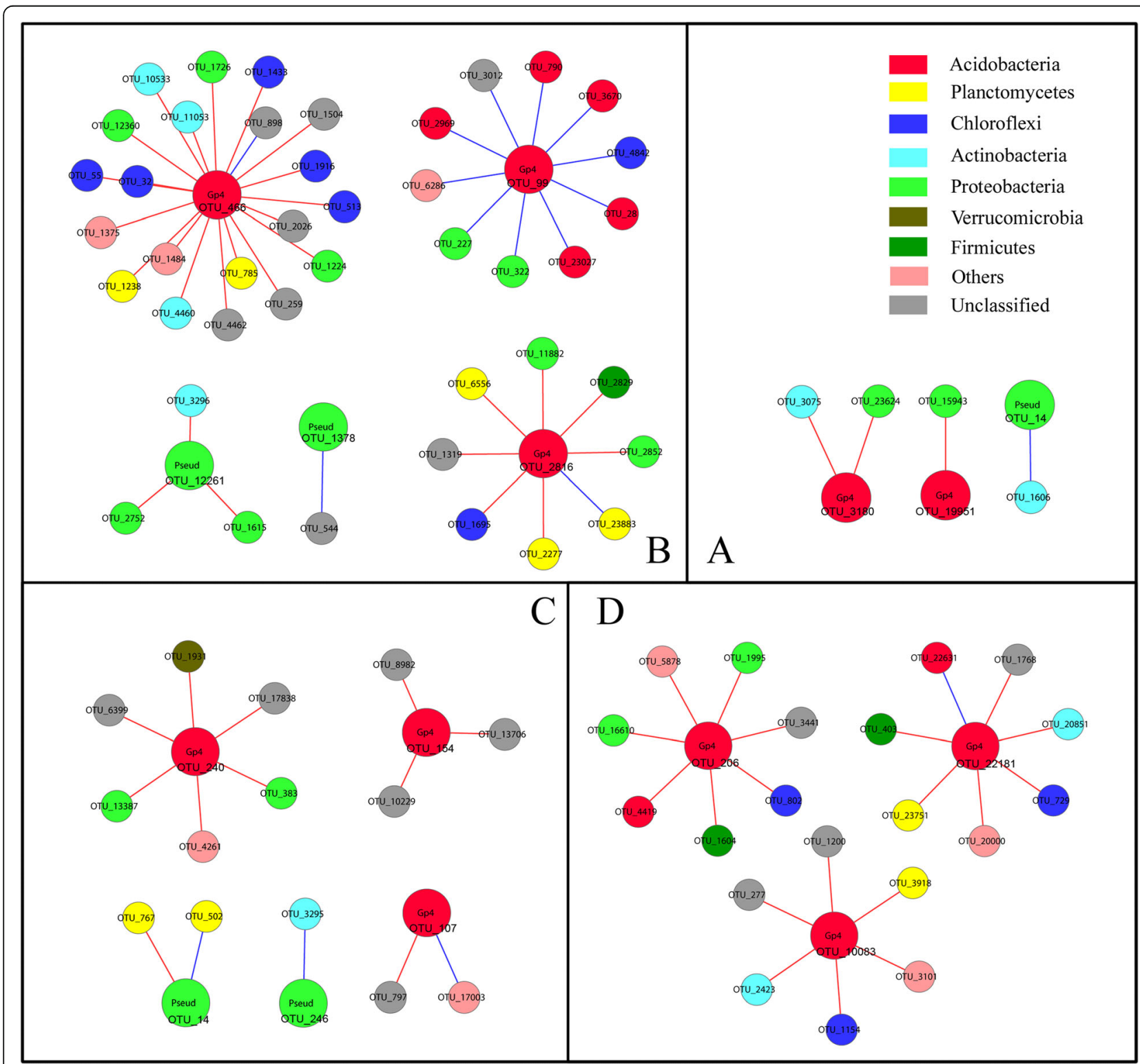

Fig. 3 Network interactions of the only OTUs of Gp4 and Pseudomonas in Control (a), MR (b), LR (c) and TR (d). Each node signifies an OTU which could correspond to a microbial population. Colors of the nodes indicate different major phylum. A blue line indicates a positive interaction between 2 individual nodes, while a red line indicates a negative interaction 
correlation with tobacco, the abundances of 3 phyla were positively correlated with tobacco disease rate, including Planctomycetes, Chloroflexi and Firmicutes. On the contrary, abundances of 8 phyla were negatively correlated with tobacco morbidity (bacterial wilt), including Acidobacteria, Verrucomicrobia, Crenarchaeota, Gemmatimonadetes, Bacteroidetes, Nitrospira, WS3, and BRC1 (Additional file 1: Table S4a). At the genus level, abundances of 7 genera (relative abundance $>0.5 \%$ ) decreased as the tobacco disease rate increased, whereas abundances of 13 genera increased with the increase of tobacco disease rate (Additional file 1: Table S4b). Especially, we found that the abundance of Pseudomonas was negatively correlated with incidence of tobacco bacterial wilt in tobacco mature period.

To reveal the relationship between microbial communities and soil properties, mantel test was conducted. Results showed that soil water content, $\mathrm{pH}$ and the content of $\mathrm{K}, \mathrm{Ca}$, and $\mathrm{Mn}$ had significant impacts on relative abundances of some microbial phyla and genera (Additional file 1: Table S5). For example, water content was correlated with the abundances of Acidobacteria, Actinobacteria, Firmicutes and Nitrospira. And soil Ca content had significant $(p<0.05)$ impacts on Acidobacteria_Gp4, Acidobacteria_Gp6 and Acidobacteria_Gp7. Furthermore, Person correlation analysis showed that the impacts were positive, and both the amount of $\mathrm{Ca}$ and relative abundances of these 3 genera were negatively correlated with tobacco disease rate.

Collectively, 2 PLSPMs were constructed to profile the relationship among soil properties, microbial communities and tobacco bacterial wilt. In the first model, the relationship among soil properties, microbial community structure, diversity and tobacco disease were explored. Results showed that soil properties had impacts on soil microbial communities; both soil properties and microbial communities influenced community diversity; and all of them contributed to tobacco bacterial wilt. All the correlations were significant $(p<0.01)$ in the model. Goodness of fit (Gof) value was 0.4599, bigger than 0.35 , indicating that the model was reliable. In order to further reveal the relationship between microbial communities of fallow period and tobacco mature period, the second model was constructed. We found that soil properties in mature period were determined by soil properties and microorganisms of fallow period. And all of them shaped microbial communities of tobacco mature period (Fig. 4). Gof was 0.4995 and all correlations were significant $(p<0.01)$, except for the link between soil properties and microbial communities of fallow period.

\section{Discussion}

Because of its value in microbial ecology and disease control of agriculture, the interaction mechanism between crop planting and soil microbial communities is of high significance in scientific research. It has been reported that plants could offer a specific environment that was selective to soil microbial communities, filtering out specific populations [29, 30]. So a general response pattern of soil microbial communities to crop planting is expected to exist. By investigating soil microbial communities during tobacco cultivation across different rotation types, we aimed to reveal the general response pattern of soil microbial communities to tobacco
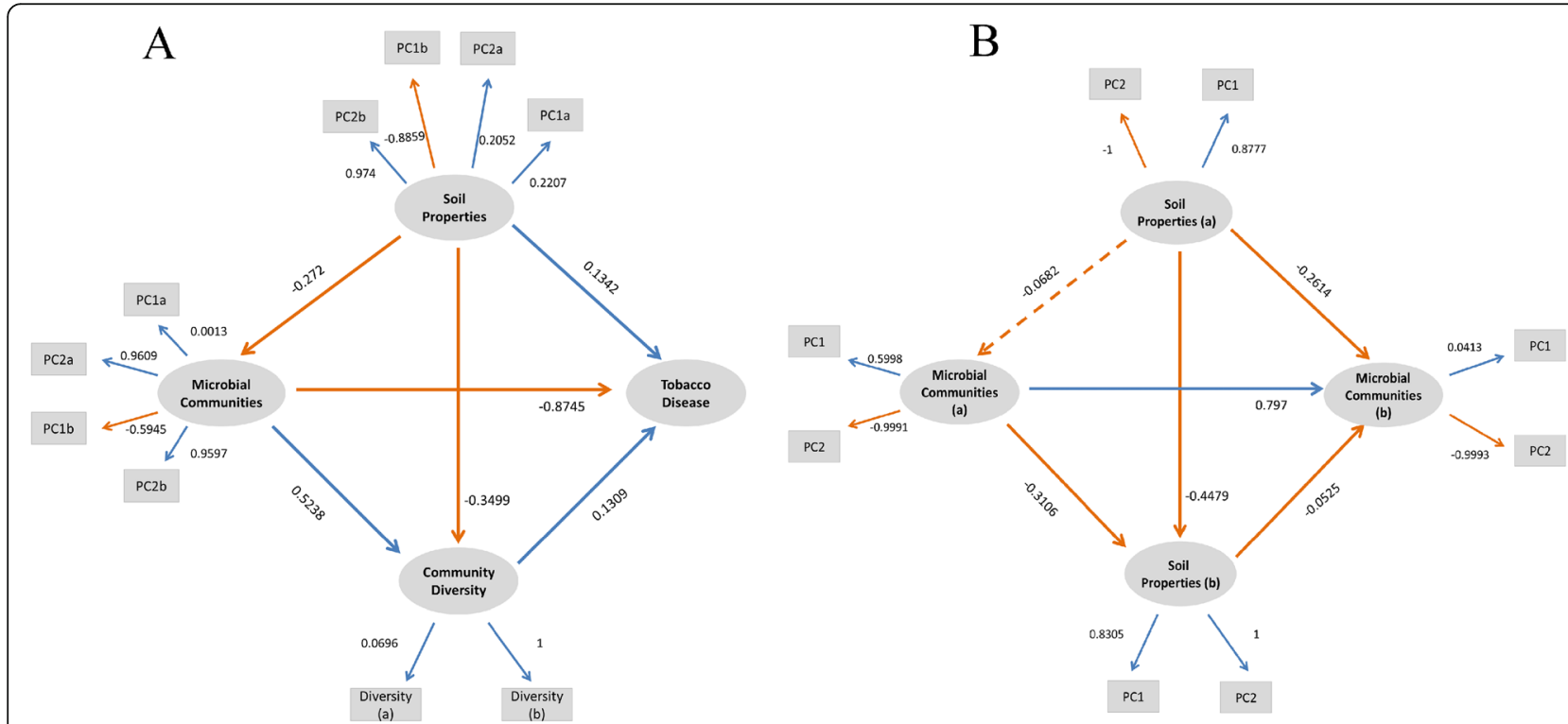

Fig. 4 Partial least squares path modeling (PLSPM) about correlations among soil properties, microbial communities, community diversity and tobacco bacterial wilt (a), as well as among soil properties, microbial communities of fallow period and tobacco mature period (b) 
cultivation and how they shaped tobacco health ultimately. Particularly, this study suggested that interactions among microbial populations also played an important role in shaping crop health.

\section{The succession of soil microbial communities during tobacco cultivation}

Soil microbial communities of tobacco mature period were shaped by that of fallow period. It was evidenced by that, most dominant microbial phyla and genera exhibited positive correlations in abundance between two periods, and no microbial genus showed negative correlation. In other word, the abundance pattern of major microbial populations in 4 groups remained the same, although their abundances changed with time. For instance, Acidobacteria was significantly more abundant in MR and less abundant in Control at fallow stage. Their relative abundances decreased at mature stage in almost all groups, but still remained more abundant in MR and less abundant in Control. In this way, we speculated that soil microbial community structure of tobacco mature stage ware mainly shaped by that of fallow stage. The result was conflict with some previous researches, which indicated that contribution of initial community to later community is usually small due to stochastic factors or changing of environmental factors [31,32]. Probably because of the specific selection of plants to communities (e.g., root exudates) [29], stochastic or environmental factors have a relatively smaller impact on succession of soil microbial communities, resulting in the significant correlation between initial and later microbial composition in this study.

Actually, plants did have certain selective pattern on soil microbes, as previous study showed that bacterial communities not only adapt to plant type, but also change over time with the same plant type [33]. Here, the abundances of Proteobacteria and Planctomycetes increased while Acidobacteria and Verrucomicrobia decreased during tobacco cultivation. It might be related to root exudates (e.g., organic acids, lactam, esters, glycerol and nicotine) also [29,34], which needs to be explored further. The impacts of root exudates on plant health related microorganisms are particularly significant. It was reported that plant root exudates could increase microbial activity in the rhizosphere, but some root exudates (e.g., phenylpropanoid) play important role in defense against non-host pathogens [34]. Pseudomonas could produce antifungal phloroglucinols in soils naturally suppressive to tobacco disease [35]. In this study, the abundance of Pseudomonas decreased in tobacco disease period while Ralstonia had no significant change in abundance between 2 periods. The result probably resulted from the different impact of tobacco root exudates on pathogens and antagonists.
Interestingly, many (not all) microbial populations which significantly correlated to tobacco disease rate, had abundance patterns shaped by that of previous period. It indicated that the abundance patterns of these potential disease inducible or suppressive species were already formed in fallow period. For instance, the relative abundance of Chloroflexi (mainly Ktedonobacter) was positively correlated with tobacco disease rate. They could be potential disease inducible microorganisms, because few plant-beneficial properties were associated with Chloroflexi. For example, they can't fix nitrogen, thus may compete for nitrogen resource with tobacco plants [36]. On the contrary, Acidobacteria (including Acidobacteria_Gp6 and Acidobacteria_Gp4) were negatively correlated with tobacco disease, suggesting their possible function in disease suppression. They had genes that encode polyketide synthase and nonribosomal peptide synthase enzymes, which well known for their roles in the synthesis of antibiotics and antifungals [18, 37]. These characteristics made most of Acidobacteria populations beneficial to tobacco health. However, both of them had abundance pattern shaped at fallow stage, suggesting the significance of initial soil microbial communities before tobacco transplanting to tobacco health.

\section{The impacts of crop rotation on microbial succession and tobacco health}

One of the major results of this study was that microbial community diversity decreased dramatically after tobacco planting but still remained relatively high in MR, which was of low tobacco disease rate. A previous study showed that microbial diversity index showed a trend of decreasing under continuous tobacco cropping [38], indicating a negative influence of tobacco on soil microbial diversity. More importantly, rotation may affect microbial community diversity reversely. In some cases, Shannon diversity under rotation cropping was significantly higher than that in continuous cropping [18, 39]. So we supposed that the maize rotation may decrease the incidence of tobacco bacterial wilt by alleviating the decrease of diversity during tobacco plantation. In other words, soil microbial communities with high diversity may have advantages in preventing plant from disease. Griffiths reported that there was no direct relationship between biodiversity and function, but the soils with the highest biodiversity were more resistant to the stress than soils with impaired biodiversity [40]. And there is evidence that soil microbial diversity confers protection against soil-borne disease, significant for agricultural sustainability [35]. Collectively, our results supported that tobacco farmlands with high biodiversity were more resistant to infection of pathogen.

Decreasing the co-occurrences/connections among microbes might be another general pattern of how soil 
microbial communities responded to tobacco planting. In this study, the number of nodes and links in pMENs of all groups decreased in tobacco mature period except for MR. A study suggested a positive correlation between biodiversity and interactions of communities, as elevated biodiversity was observed in more connected communities [41]. Consistent with the previous study, MR microbial communities had higher diversity and larger ecological network. There were extensive cooperation and competition among different populations, and the maintaining of such relationships makes community a homeostatic systems [42]. If the balance is destroyed by environments or invader, certain species of the community will prosper or decline even extinct [43]. In this study the decrease of co-occurrence among microbial populations might result from/in prosper of certain species, destroyed biodiversity and destruction of balance. Therefore, the impaired interactions among microbial populations might make communities more susceptible to invasion of pathogens.

Further analyses indicated that there was stronger competition between potential disease suppressive (e.g., Acidobacteria) and inducible bacteria (e.g., Chloroflexi) in maize rotation systems. Previous studies showed that some bacteria could protect the plant directly by producing antibacterial agent, or indirectly by enhancing rhizosphere function of antagonistic populations [8]. Here, Acidobacteria_Gp4 had more negative links with disease inducible bacteria (e.g., Chloroflexi and Planctomycetes), and more positive links with disease suppressive populations (e.g., Acidobacteria). It indicated that Gp4 enhanced their cooperation with probiotic bacteria and competition with potential pathogens in MR. Pathogenesis is a process in which pathogens and biocontrol agents compete with each other, supporting our results [4]. The enhanced interactions limited the prosperity of Raltonia and other potential pathogens, making maize rotation an effective system in controlling tobacco bacterial wilt. In conclusion, our results indicated that plant disease resulted largely from the interactions among different microbial populations, particularly between pathogens and probiotic bacteria, although further experimental verifications are necessary.

Finally, we conducted PLSPM analyses to profile the complex interactions among soil properties, microbial communities and tobacco health. On the one hand, soil properties could affect tobacco directly. For example, Ca is not only a necessary element for plant growth, it could also increase tobacco's resistance to pathogen indirectly. Moshe Sagi found that the plant homolog can be stimulated directly by $\mathrm{Ca}_{2}^{+}$to produce $\mathrm{O}_{2}^{-}$, which was considered to be a component of the resistance response of plants to pathogen challenge [44]. It supported our result that the amount of $\mathrm{Ca}$ in soil was negatively correlated with tobacco morbidity. On the other hand, soil properties could affect tobacco health indirectly through soil microbial communities. Noah Fierer and Robert B. Jackson reported the diversity and richness of soil bacterial communities could largely be explained by soil $\mathrm{pH}[6]$. In this study, soil $\mathrm{pH}(5.5 \sim 6.5)$ and water content (15\% $25 \%$ ) were suitable for tobacco plantation. They were different in 4 rotation groups or between 2 periods, indicating their impacts on soil microbial communities, consistent with previous study. Then, soil microorganisms impact tobacco health in many ways. Some of the indigenous microorganisms protect susceptible crops from certain pathogens, whereas disease-conducive microorganisms infect plants or permit spread of the pathogens [8, 45]. By studying the shift of soil microorganisms in composition, structure and co-occurrence during tobacco planting across different rotation types, our research profiled a general picture of the succession pattern of microbial communities and its relationship with tobacco health.

\section{Conclusions}

In summary, we found that (i) both soil microbial communities of fallow stage and tobacco selection shaped the communities of tobacco mature stage; (ii) effective rotation crop (maize) could decrease the incidence of tobacco bacterial wilt by alleviating the decrease in diversity and co-occurrences of microbial populations.

\section{Additional file}

Additional file 1 Figure S1. Rarefaction curves of 16r RNA gene sequencing data. Figure S2. Composition and structure of soil microbial communities in each group. Figure S3. Phylogenetic molecular ecological networks (pMEN) of microbial communities in each group, and number of nodes and links of each pMEN. Table S1. Soil properties. Table S2(a). Correlation of microbial populations in abundance between two periods at the phylum level. (b). Correlation of microbial populations in abundance between two periods at the genus level. Table S3.

Topological properties of the empirical pMENs of microbial communities in eight groups. Table S4(a). Correlation between abundance of microbial populations and tobacco morbidity at the phylum level. (b). Correlation between abundance of microbial populations and tobacco morbidity at the genus level. Table S5(a). Mantel test of sequencing data with environmental attributes at the phylum level. (b). Mantel test of sequencing data with environmental attributes at the genus level. (DOCX $1100 \mathrm{~kb})$

\section{Acknowledgements}

This research was supported by Hunan Branch of China National Tobacco Corporation (CYKJ2014-05).

\section{Authors' contributions}

JN and ZR: Data analysis and manuscript preparation. LD and HY: Design and conception of the experiments. ZR, CZ and JN: Execution of the experiments. FT, HY and LD: Participated in discussions and supervised overall experimental and theoretical works. All authors have read and approved the manuscript. 


\section{Competing interests}

The authors declare that they have no competing interests.

\section{Author details}

'School of Minerals Processing and Bioengineering, Central South University, Changsha 410083, China. ${ }^{2}$ Key laboratory of Biometallurgy, Ministry of Education, Changsha 410083, China. ${ }^{3}$ College of agronomy, Hunan Agricultural University, Changsha 410128, China. ${ }^{4}$ Tobacco monopoly bureau of Xiangxi Autonomous Prefecture, Hunan, Jishou 416000, China.

\section{Received: 7 February 2016 Accepted: 21 September 2016} Published online: 06 October 2016

\section{References}

1. Van Der Heijden MG, Bardgett RD, Van Straalen NM. The unseen majority: soil microbes as drivers of plant diversity and productivity in terrestrial ecosystems. Ecol Lett. 2008;11(3):296-310.

2. Burdon J, Silk J. Sources and patterns of diversity in plant-pathogenic fungi. Phytopathology. 1997:87(7):664-9

3. Haas D, Défago G. Biological control of soil-borne pathogens by fluorescent pseudomonads. Nat Rev Microbiol. 2005;3(4):307-19.

4. Paulitz T. Population dynamics of biocontrol agents and pathogens in soils and rhizospheres. Eur J Plant Pathol. 2000;106(5):401-13.

5. Griffiths RI, Thomson BC, James P, Bell T, Bailey M, Whiteley AS. The bacterial biogeography of British soils. Environ Microbiol. 2011;13(6):1642-54.

6. Fierer $\mathrm{N}$, Jackson RB. The diversity and biogeography of soil bacterial communities. Proc Natl Acad Sci U S A. 2006;103(3):626-31. Epub 2006/01/13.

7. Zhang $B$, He $H$, Ding $X$, Zhang $X$, Zhang $X$, Yang $X$, et al. Soil microbial community dynamics over a maize (Zea mays L.) growing season under conventional- and no-tillage practices in a rainfed agroecosystem. Soil Tillage Res. 2012;124:153-60.

8. Zhang Y, Liu G, Ye X, Li Y, He C, Li W, et al. Changes of soil enzyme activities and microorganism amounts at different growing stages of fluecured tobacco. Soils. 2010;42(1):39-44

9. Zhang J, Guo H, Li L, Duan F, Guo C, Zhou J. Research on dynamic change of tobacco soil microbes in Dali. Acta Tabacaria Sinica. 2010;16(5):55.

10. Montoya JM, Pimm SL, Solé RV. Ecological networks and their fragility. Nature. 2006;442(7100):259-64.

11. Jin F, Ding Y, Ding W, Reddy MS, Fernando WG, Du B. Genetic diversity and phylogeny of antagonistic bacteria against Phytophthora nicotianae isolated from tobacco rhizosphere. Int J Mol Sci. 2011;12(5):3055-71.

12. Troxler J, Berling CH, Moe"Nne-Loccoz Y, Keel C, DÉFago G. Interactions between the biocontrol agent Pseudomonas fluorescens CHAO and Thielaviopsis basicola in tobacco roots observed by immunofluorescence microscopy. Plant Pathol. 1997:46(1):62-71.

13. Zhou J, Deng Y, Luo F, He Z, Yang Y. Phylogenetic molecular ecological network of soil microbial communities in response to elevated CO2. MBio. 2011;2(4). Epub 2011/07/28

14. Zhou J, Deng Y, Luo F, He Z, Tu Q, Zhi X. Functional molecular ecological networks. mBio. 2010;1(4):doi: 10.1128/mBio.00169-10.

15. Zhang $X$, Pan Z, Zhou X, Wuzhong N. Autotoxicity and continuous cropping obstacles: a review. Chin J Soil Sci. 2007:38(4):781-4.

16. Kincaid RR. Crop rotation and fallowing in relation to tobacco disease control. Bot Rev. 1960;26(2):261-76

17. Jiguang Z, Linlin Z, Yi S, Zhongfeng Z, Xinghua M, Guoming S, et al. Effects of different planting patterns on soil microbial community, yield and quality of flue-cured tobacco leaves. Trans Chin Soc Agric Eng. 2012;28(19):93-102.

18. Niu J, Chao J, Xiao Y, Chen W, Zhang C, Liu X, et al. Insight into the effects of different cropping systems on soil bacterial community and tobacco bacterial wilt rate. J Basic Microbiol. 2016:doi: 10.1002/jobm.201600222.

19. CLARKE KR. Non-parametric multivariate analyses of changes in community structure. Aust J Ecol. 1993:18:117-43.

20. Calinski. Principles and procedures of statistics - a biometrical Approach, 2nd edition - steel, RGD, Torrie, JH. Biometrics. 1981:37:859-60.

21. Smouse PE, Long JC, Sokal RR. Multiple regression and correlation extensions of the Mantel test of matrix correspondence. Syst Zool. 1986; 35(4):627-32.

22. Statistical Package R. R: A language of environment for statistical computing. Vienna, Austria: R Foundation for Statistical Computing; 2009.
23. Luo F, Yang Y, Zhong J, Gao H, Khan L, Thompson DK, et al. Constructing gene co-expression networks and predicting functions of unknown genes by random matrix theory. BMC Bioinformatics. 2007;8:299. Epub 2007/08/19.

24. Horvath S, Dong J. Geometric interpretation of gene coexpression network analysis. PLoS Comput Biol. 2008;4(8):e1000117.

25. Horvath S, Zhang B, Carlson M, Lu K, Zhu S, Felciano R, et al. Analysis of oncogenic signaling networks in glioblastoma identifies ASPM as a molecular target. Proc Natl Acad Sci. 2006;103(46):17402-7.

26. Luo F, Zhong JX, Yang YF, Scheuermann RH, Zhou AJZ. Application of random matrix theory to biological networks. Phys Lett A. 2006;357:420-3.

27. Yin H, Niu J, Ren Y, Cong J, Zhang X, Fan F, et al. An integrated insight into the response of sedimentary microbial communities to heavy metal contamination. Sci Rep. 2015:5:14266.

28. Cline MS, Smoot M, Cerami E, Kuchinsky A, Landys N, Workman C, et al. Integration of biological networks and gene expression data using cytoscape. Nat Protoc. 2007;2:2366-82.

29. Hartmann A, Schmid M, Tuinen D, Berg G. Plant-driven selection of microbes. Plant Soil. 2008:321(1-2):235-57.

30. Hardoim PR, Andreote FD, Reinhold-Hurek B, Sessitsch A, van Overbeek LS, van Elsas JD. Rice root-associated bacteria: insights into community structures across 10 cultivars. FEMS Microbiol Ecol. 2011;77(1):154-64.

31. Lauber CL, Ramirez KS, Aanderud Z, Lennon J, Fierer N. Temporal variability in soil microbial communities across land-use types. ISME J. 2013;7(8):1641-50.

32. Zhou J, Deng Y, Zhang P, Xue K, Liang Y, Van Nostrand JD, et al. Stochasticity, succession, and environmental perturbations in a fluidic ecosystem. Proc Natl Acad Sci U S A. 2014;111(9):E836-45.

33. Garbeva P, Van Veen J, Van Elsas J. Microbial diversity in soil: selection of microbial populations by plant and soil type and implications for disease suppressiveness. Annu Rev Phytopathol. 2004;42:243-70.

34. Bais HP, Weir TL, Perry LG, Gilroy S, Vivanco JM. The role of root exudates in rhizosphere interactions with plants and other organisms. Annu Rev Plant Biol. 2006;57:233-66

35. Brussaard L, De Ruiter PC, Brown GG. Soil biodiversity for agricultural sustainability. Agric Ecosyst Environ. 2007;121(3):233-44.

36. Kragelund C, Levantesi C, Borger A, Thelen K, Eikelboom D, Tandoi V, et al. Identity, abundance and ecophysiology of filamentous Chloroflexi species present in activated sludge treatment plants. FEMS Microbiol Ecol. 2007; 59(3):671-82. Epub 2007/03/27

37. Ward NL, Challacombe JF, Janssen PH, Henrissat B, Coutinho PM, Wu M, et al. Three genomes from the phylum Acidobacteria provide insight into the lifestyles of these microorganisms in soils. Appl Environ Microbiol. 2009; 75(7):2046-56. Epub 2009/02/10

38. Li G, Xue-long W, Yong-de C, Yu-gang Z. Effects of continuous tobacco cropping on the microbes in tobacco soil. Hubei Agric Sci. 2009;48(10): 2444-5.

39. Fang S-M, Tang L-N, Chen S-H, Gu G, Chen Y-S. Influence of crop rotation on tobacco bacterial wilt number and pothogenesy. Chin J Eco-Agric. 2011; 19(2):377-82.

40. Griffiths BS, Ritz K, Bardgett RD, Cook R, Christensen S, Ekelund F, et al. Ecosystem response of pasture soil communities to fumigation-induced microbial diversity reductions: an examination of the biodiversity-ecosystem function relationship. Oikos. 2000;90(2):279-94.

41. Widder S, Besemer K, Singer GA, Ceola S, Bertuzzo E, Quince C, et al. Fluvial network organization imprints on microbial co-occurrence networks. Proc Natl Acad Sci. 2014;111(35):12799-804.

42. McCann K, Hastings A, Huxel GR. Weak trophic interactions and the balance of nature. Nature. 1998:395(6704):794-8.

43. Tilman D. Niche tradeoffs, neutrality, and community structure: a stochastic theory of resource competition, invasion, and community assembly. Proc Natl Acad Sci U S A. 2004;101(30):10854-61

44. Sagi M, Fluhr R. Superoxide production by plant homologues of the gp91phox NADPH oxidase. Modulation of activity by calcium and by tobacco mosaic virus infection. Plant Physiol. 2001;126(3):1281-90.

45. Kyselkova M, Kopecky J, Frapolli M, Defago G, Sagova-Mareckova M Grundmann $\mathrm{GL}$, et al. Comparison of rhizobacterial community composition in soil suppressive or conducive to tobacco black root rot disease. ISME J. 2009;3(10):1127-38. Epub 2009/06/26 